\title{
Low-Voltage Distribution Network Reconfiguration Considering High Penetration of Electric Vehicles - A UK Case Study
}

\author{
Zhi Qiao \\ School of Engineering and Applied Science \\ Aston University \\ Birmingham, United Kingdom \\ qiaoz@aston.ac.uk
}

\author{
Jin Yang \\ School of Engineering and Applied Science \\ Aston University \\ Birmingham, United Kingdom \\ j.yang8@aston.ac.uk
}

\begin{abstract}
Network reconfiguration is one of the electricity network optimization techniques for losses reduction and load balancing by changing the open-closed status of sectionalized switches. In this paper, an application of network reconfiguration in low-voltage (LV) domestic distribution network with high penetration of electric vehicles (EVs) is presented. Firstly, high resolution time-series domestic load profiles are generated and validated with measured data at substation. Electric vehicle charging profiles at both fast and slow charging modes are modelled and produced. Then the impact of increasing penetration of EVs on the network thermal and voltage constraints is quantified. To optimize the network topology, suitable locations of newly-installed switches and their operational time are identified by branch-exchange method and exhaustive search algorithm (ESA) due to the small size of network and less potential location. The proposed approach is applied to two practical $400 \mathrm{~V} \mathrm{~V}$ distribution networks interconnected by underground linkboxes which are used to provide back-feeds during routine maintenances or emergency conditions. By adding new sectionalized switches, the $L V$ network will obtain more operational flexibility and could better explore its potential for further study under Smart Grid scenarios.
\end{abstract}

Keywords-network reconfiguration, distribution networks, loss reduction, electric vehicles

\section{INTRODUCTION}

A series of low carbon policies are deployed by the UK government to reflect on the growing concerns on carbon emission and greenhouse effect - so-called "low carbon" strategies or techniques. These measures include incorporating as much as possible renewable energy sources (RESs) [1], utilizing more electric devices such as electric vehicles (EVs) [2], particularly on domestic customers' side to urge clean electricity generation and "green consumption" in low-voltage (LV) distribution networks. Moreover, Economy 7 and Smart Meters are introduced to domestic users by the Distribution Network Operators (DNOs) to encourage customers towards a more economical consumption behavior. The embedding of EVs increases the overall demand on the distribution networks, especially in peak hours, which could cause power congestion and voltage issues. Therefore, as the forefront link between utility and customers, LV distribution networks need to be modified, or even upgraded, to face these challenges. A practical and economical approach is required to mitigate potential impacts on networks or enhance network capability without substantial reinforcement.

Various techniques are proposed including installation of centralized/decentralized energy storage systems (ESSs), battery management systems (BMSs) [3], vehicle-to-grid (V2G) technology with charging/discharging management system[4], demand-side management (DSM) techniques [5]. Among these candidate solutions, dedicated equipment, such as central control unit, extensive measurement/monitoring and communication infrastructure are required to achieve proposed functions. However, these measures introduce huge capital costs to either customers or DNOs if considered to be rolled-out.

The concept of network reconfiguration has been mainly used for medium voltage (MV) distribution network optimization, by changing the open-closed status of tie switches or sectionalizing switches to alter the topology of network in order to shift heavy load to adjacent branches or substations [6], also known as automatic load transferring (ALT). By doing so, thermal and voltage issues could be mitigated to some extent. Unlike MV distribution networks which have a meshed topology and operate in open-ring mode, the LV distribution system cannot be reconfigured into mesh connection without adding extra cable routes. In the UK, underground linkboxes are used to provide back-feeding flexibility to transfer loads when faults/emergency conditions occur along a long radial feeder, to be connected to the adjacent feeder, or under routine maintenance schedules, to minimize the number of lost customers, hence customer interruptions (CIs) and customer minute lost (CML), which are important indices to measure the DNO's supply quality. These interconnection devices are old solid electricity switching device which has no metering and automation functions. To the authors view, by replacing them with remote-controlled circuit breakers (CBs) and installing additional $\mathrm{CBs}$ will enable $\mathrm{LV}$ networks for more options to reconfigure. Thus by properly opening or closing these $\mathrm{CBs}$, the boundaries among distribution feeders can be modified to achieve more flexible operational configuration.

Based on the above assumption, this paper introduces a procedure for $\mathrm{LV}$ network reconfiguration with high penetration of EVs. Objectives considered are network loss reduction, power quality improvement, e.g. voltage issues, and network capacity, i.e. thermal constraints. Practical networks are modelled using 
OpenDSS (Distribution System Simulator) [7] with optimization algorithm interfaced by MATLAB codes.

This paper is organized as follows. Section II summarizes the mathematical problem and published optimization approaches. Section III describes system modelling and all the input data including LV networks, load profiles and EV charging profiles. In Section IV, the impact of EV charging on network is quantified at increasing penetration levels respectively. In Section IV, branch exchange method and exhaustive search algorithm is adopted to identify the suitable location of installing new switches and their operational time and frequency, with discussions about this method and applications. Conclusions and further works are drawn in Section V.

\section{STATE-OF-THE-ART}

Branch exchange is a classic method by connecting the tie line and opening some other branch to restore a radial structure for all the possible condition to identify global optimal configuration. Heuristic search approaches (HAS) are more efficient to find suboptimal network configuration [9]. Single loop optimization method is connecting one branch to form a meshed configuration and then opening one branch in this loop each time to bring back its radial character for searching ideal configuration [10]. Decentralized approach in [8, 11] is a combination of the two method mentioned above to improve the efficiency. Intelligent approaches are also suggested to reduce calculation time from the algorithm aspect in literature, such as ant colony search algorithm [12], genetic algorithm [13]. They are advantageous in dealing with complex meshed networks, multi-objective functions or large-scale time-series optimization problems. In this paper, branch exchange method is implemented for the reason that the topology of LV networks are radial in nature. In addition, compared to MV networks, there are low number of LV tie switches and branches that can be operated to find the optimal configuration. Additional CBs have to be installed to enable more optimization options. Both their locations and time of operations provide higher flexibility than the conventional linkboxes. Exhaust search algorithm is engaged in this study to identify time-series global optimal configuration for illustration the benefit of $400 \mathrm{~V}$ distribution network reconfiguration.

\section{NETWORK MODELLING AND DATA}

Detailed modelling of LV distribution network has not been extensively covered in published researches. In this section, very detailed models up to the service cable level are introduced. Load profiles employed are validated with real-time monitoring data. EV charging profiles are compared with the result in literature. These provide good guidelines for detailed modelling of distribution networks.

\section{A. Network Description and Models}

Two real 400V (230V single-phase) LV residential distribution networks located in Midlands of England are used in this case study. LV Network1 is supplied by a $500 \mathrm{kVA}$ distribution transformer with 292 domestic customers. Network2 is supplied by an $800 \mathrm{kVA}$ distribution transformer with 339 domestic customers and two primary schools. Fig. 1 shows the schematic of the two networks - blocks are used to indicate the positions of transformers, locations of two linkboxes are highlighted with underlines.

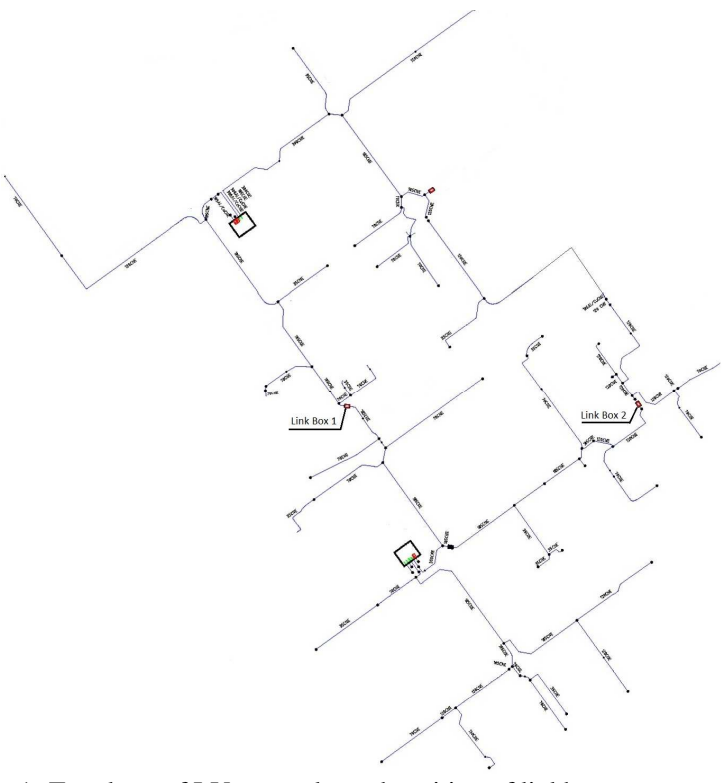

Fig. 1. Topology of LV networks and position of linkboxes

\section{B. Domestic Load Profiles}

Load profiles are essential in obtaining valid results. In LV networks, majority of customer loads are not monitored, even those customers with smart meters installed, the sampling frequency is low (every 30min or lower) and confidential.

Alternative solution proposed in [14], known as CREST (Centre for Renewable Energy Systems Technology) tool, using residents electricity consumption behaviors and electric appliance rated power to model the power consumption of a dwelling in 1-min resolution. This model is implemented to simulate the customers in these two networks. All domestic users are modelled as non-electric central heating residential loads (i.e. gas heating available in the dwelling), with an ADMD (After Diversity Maximum Demand) of $2 \mathrm{~kW}$. Driven by MATLAB codes, the CREST tool [14] is used to create 1000 independent load profiles with random numbers of residents, active people profiles, household appliance and usage profile.

For network one, 292 load profiles are selected randomly in the pool of 1000 independent load profiles and accumulated load profile are compared with the measured data at substations. Two parameters, covariance and correlation coefficient are implemented to evaluate how the accumulated load shape and the loads measured at substation related. Due to the load measured at substation is in $10 \mathrm{~min}$ resolution, synthetic load profile is converted to $10 \mathrm{~min}$ resolution by mean value for comparison. The formula for calculating covariance of these two group of data is shown below:

$$
\operatorname{Cov}(x, y)=\frac{\sum_{i=1}^{n}\left(x_{i}-\bar{x}\right)\left(y_{i}-\bar{y}\right)}{n-1}
$$

Correlation coefficient is defined as:

$$
\operatorname{Corr}(x, y)=r_{x y} \frac{1}{n} \sum_{i=1}^{n}\left(\frac{x_{i}-\bar{x}}{s_{x}}\right)\left(\frac{y_{i}-\bar{y}}{s_{y}}\right)
$$


where $\bar{x}$ and $\bar{y}$ are the mean values of measured data and accumulated synthetic data, $s_{x}$ and $s_{y}$ are standard deviation of the corresponding data and $\mathrm{n}$ is the number of points, as 144 (sampling in 10-min resolution) is this study.

In Network1, one set of synthetic data composed of 292 individual load profiles is selected which yields largest covariance to match the measured data shown in Fig. 2. Correlation coefficient of these two groups of data is 0.9041 , which indicate these two group of data are highly related.

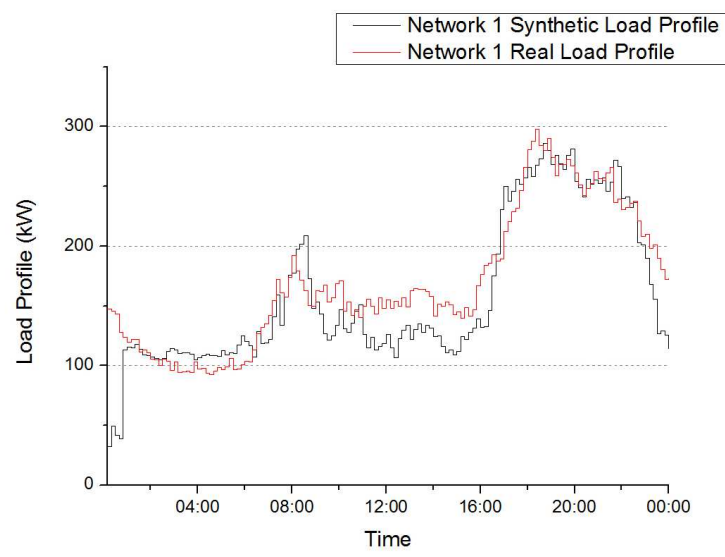

Fig. 2. Network1 synthetic load profile compared with measured data.

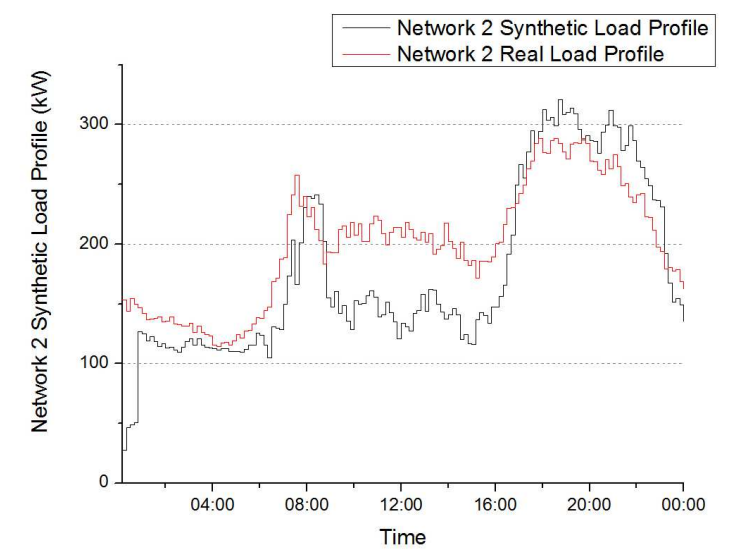

Fig. 3. Network2 synthetic load profile compared with measured data.

Same process is applied for Network 2 with 339 customers and 2 primary schools. Comparison of overall synthetic load profile and measured load profile at substation is shown in Fig. 3 . Correlation coefficient of these two groups of data is 0.8584 .

Unlike the result simulated in network 1, two groups of loads are is much closed during the day, there is a clear difference between 9 am to $4 \mathrm{pm}$ in Fig.3. This can caused by the power consumption of two primary schools. Since no clear model of small commercials or public facilities, or measured data available, here the author assume these two primary schools as constant loads and they are at the same size.

Average value of the difference between $8: 40$ to $16: 30$ is calculated and converted to $1 \mathrm{~min}$ resolution. The constant load during this period is $33.0 \mathrm{~kW}$ for each primary school. As presented in Fig. 4, the converted synthetic overall load profile is compared with the real load profile of this network again. The correlation coefficient of these two groups of data is 0.9235 after the loads of two schools considered.

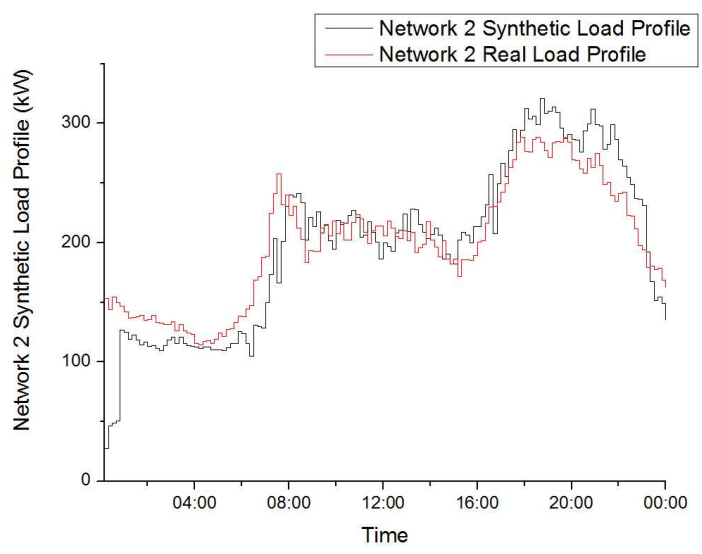

Fig. 4. converted synthetic overall load profile compared with measured data.

These figures and correlation coefficient indicate that the synthetic load profiles is reasonable and have overall similarity to the actual loading of the network. However, it is obvious that from 0:00 to 0:40 for Fig 2-4, the synthetic load profile is under estimate the power usage due to the probability function used in CREST model [14]. This phenomenon can be also obtain by using other sets of measured data. When this model [14] is used in EV charging management and demand side management study, the difference will have negative impact on the result when it is focused on shifting manageable load to off-peak time or mitigate the overall load, which will give incorrect control parameters and overestimate the benefit of algorithm. Other factors such as road lighting and public facility power consumptions are not included in the model therefore the synthetic load profile will be different from measured values at the substation..

\section{EV Charging Profiles}

To model and quantify the impact of EV charging on distribution networks, EV charging profiles in time series of high resolution are required. Here they are created and compared with charging behavior published in literature. In [15], statistical analysis of EV charging behavior in the UK is presented. As the Probability Distribution Function (PDF) includes number of connections per day, start charging time of each connection, initial and final state-of-charge (SOC) of the car battery. Nissan LEAF [16] is used for modelling the PDF from extensive monitoring data. These EVs are using 24kWh battery, composed by 12 battery unit, and charging connected to conventional mains supply at $3.6 \mathrm{~kW}$. However, Nissan also provide charging unit installed in the dwelling, which rated at $6.6 \mathrm{~kW}$. It is worth noting that the home charging unit can be operated by remote control via smartphone to take advantage of off-peak electricity rates such as Economy 7 meter tariff scheme. An assumption is made upon the model presented in [15] that if the charging power raise from $3.6 \mathrm{~kW}$ to $6.6 \mathrm{~kW}$, charging time will decrease from $40 \mathrm{~min}$ per battery unit to 22 min per battery unit without affecting start charging time, initial SOC and final SOC of each charging. For sure that if the charging power increased and charging time keep 
constant, final SOC of each charging will higher than charging at low power. The reason to ignore this fact is to keep the electricity consumption by EV charging at same level.

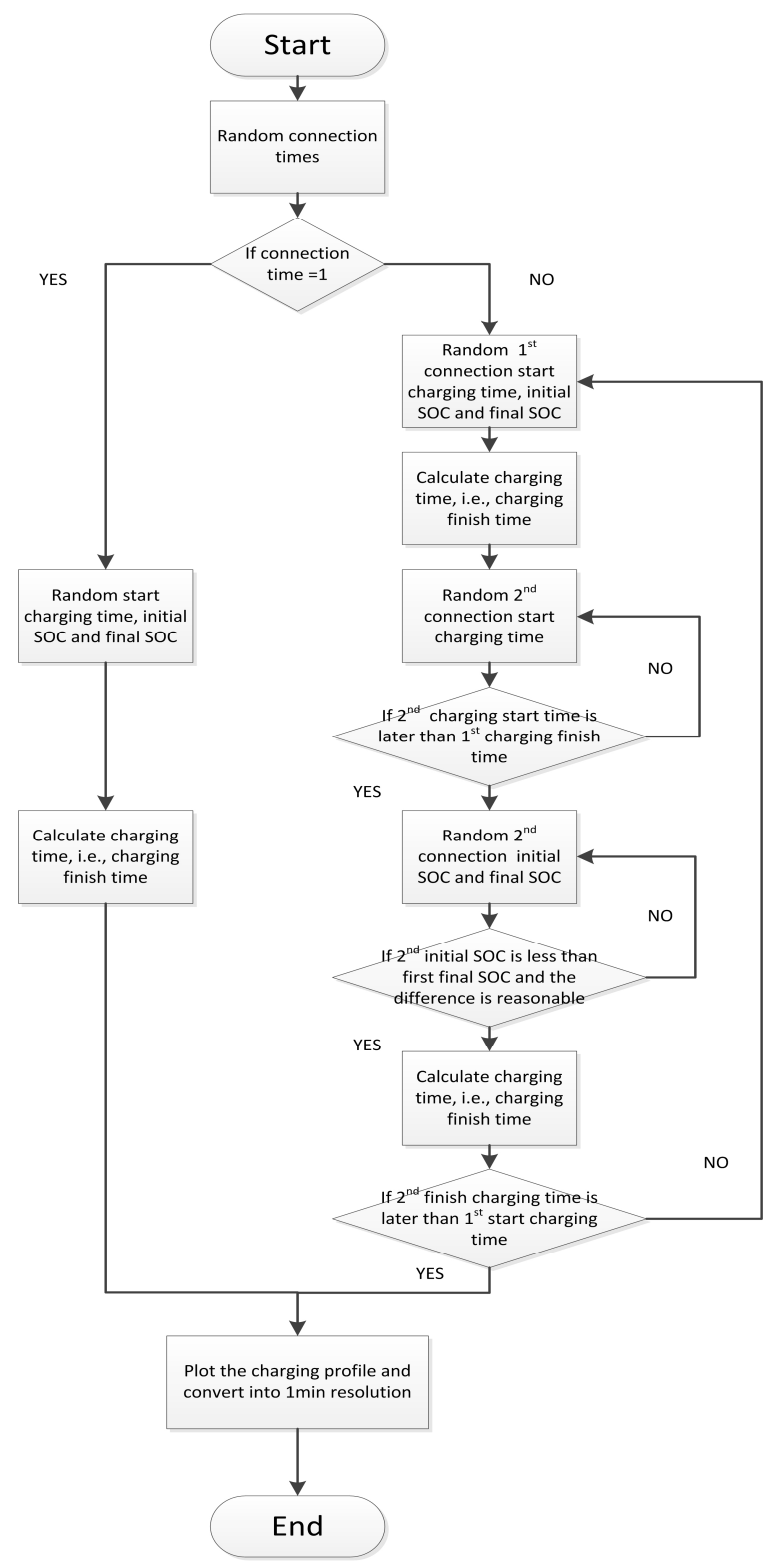

Fig. 5. EV charging profile generation flow chart.

Another factor taken into consideration is EV battery consumption for charging profile with 2 connections per day. The differential between first charging final SOC and second connection initial SOC and should within a reasonable range. As range provided in Nissan official website, LEAF $24 \mathrm{kWh}$ can travel up to 124 miles obtained from NEDC test result which may vary due to driving style, road condition and other factors. Considering an extreme condition that EV is travelling at national speed for signal carriage way which is 60 mile per hour, i.e. 1 battery unit out of 12 for every 10 mins. Based on this assumption, the battery consumption should between none to 1 battery unit out of 12 for every 10 mins. These constrains make the EV charging profile reasonable.
Two sets of charging profiles at different charging modes are created and converted into 1-min resolution, same resolution as domestic load profile. The process of generate a charging profile can be concluded as follows: Step 1 - Random connection times (once or twice charging(s) a day); Step 2 - random $1^{\text {st }}$ connection start time, initial SOC and final SOC, if this is a one connection charging, save this profile; Step 3 - if this a 2-connection charging, random $2^{\text {nd }}$ connection start time, initial SOC and final SOC, if the interval between first and second charging is reasonable, save this profile; if not, repeat Step 2. The complete process is shown in Fig. 5.

An example of EV charging profiles are plotted in Fig. 6 to better illustrate this model. It is a typical charging profile with two connection in a day. The first charging starts at 11:15 when the initial SOC is 7 battery unites out of 12 unites and ends with final SOC of 9 units. The red curve shows the charging profile charging at $3.6 \mathrm{~kW}$ and black curve indicate charging at $6.6 \mathrm{~kW}$. 2 battery unit charged during this connection. Second connection starts at 19:45 with initial SOC is 7 battery unites and ends fully charged. Slow charging cost 6 hours and 40 mins and fast charging spend 3hours 40 mins. Due to part of second charging is during the night in slow mode, this part of profile is shifted to the morning.

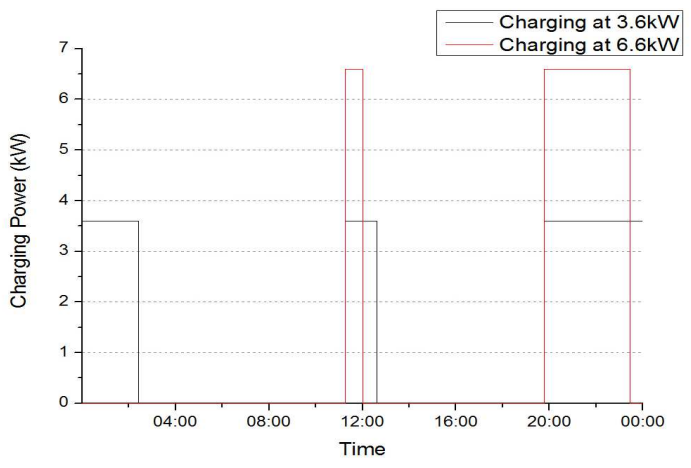

Fig. 6. example of EV charing profiles.

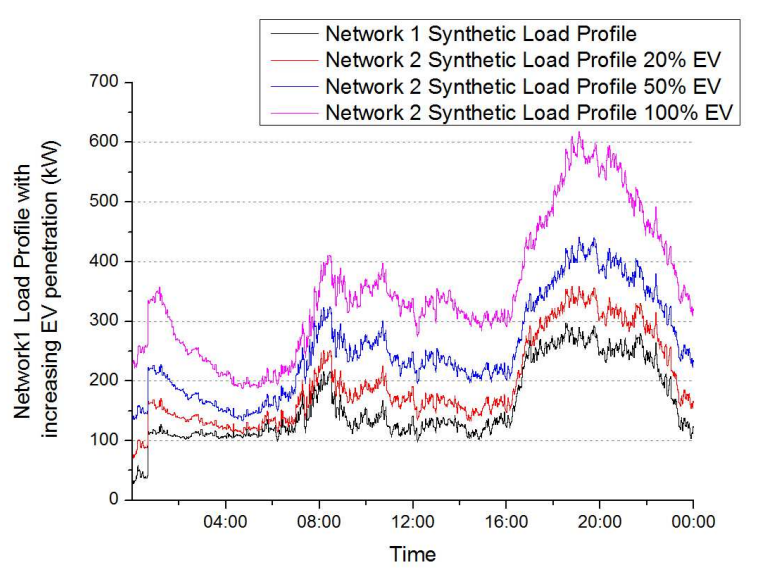

Fig. 7. Overall residential load and EV charging profile with growing penetration at slow charging mode $(3.6 \mathrm{~kW})$.

The overall network 1 residential load and EV charging profiles with growing penetration of EVs assume all the EVs are charging at slow mode are shown in Fig 7. 
If all the EV users have charging unit installed in their garages for charging at fast mode, $6.6 \mathrm{~kW}$, it will cause higher load demand in each network. Overall residential loads and EV charging at different EVs penetration are shown in Fig 8.

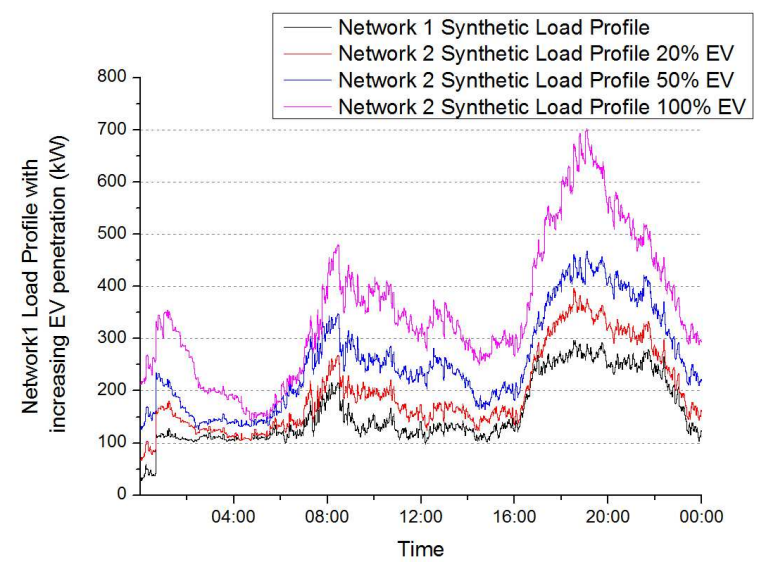

Fig. 8. Overall residential load and EV charging profile with growing penetration at fast charging mode $(6.6 \mathrm{~kW})$.

\section{Newtork Simulation System}

Open Distribution System Simulator (OpenDSS) is an open source software for electric distribution systems simulation. MATLAB is used to drive OpenDSS by in-process COM server and control the planning and analysis for target distribution systems. Fig. 9 shows an overall flow chart of the implemented simulation system.

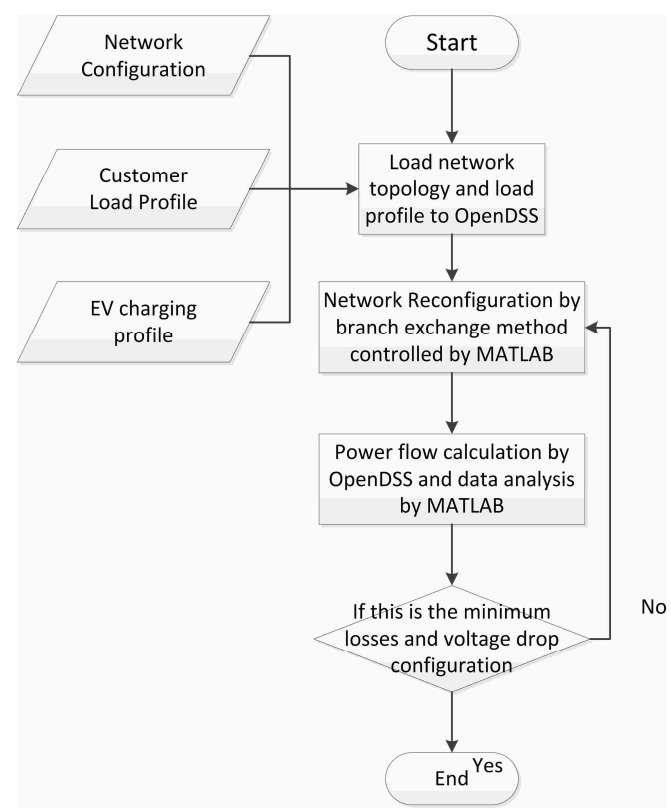

Fig. 9. Overall flow chart of the implemented simulation system.

\section{EV IMPACT RESULTS AND SOLUTION DISCUSSIONS}

In this section, losses, thermal and voltage issue is quantified by a set of load flow calculation considered increasing penetration levels of EVs. Results are obtained by using the data acquired in Section III. Graphical load flows at any one snapshot in this time-series simulation for 24 hours in 1-minute resolution can be produced. Fig. 10 shows graphical load flow (without EVs) at one snapshot (18:00 on a weekday in February 2015). Corresponding voltage profile is show is Fig. 11. 1.05 p.u. is maintained at the substation transformer secondary side to avoid low voltage at the end of this feeder. For this loading point, voltage at all nodes are well within the regulated range in the UK $(+10 \%,-6 \%$ of the nominal voltage).

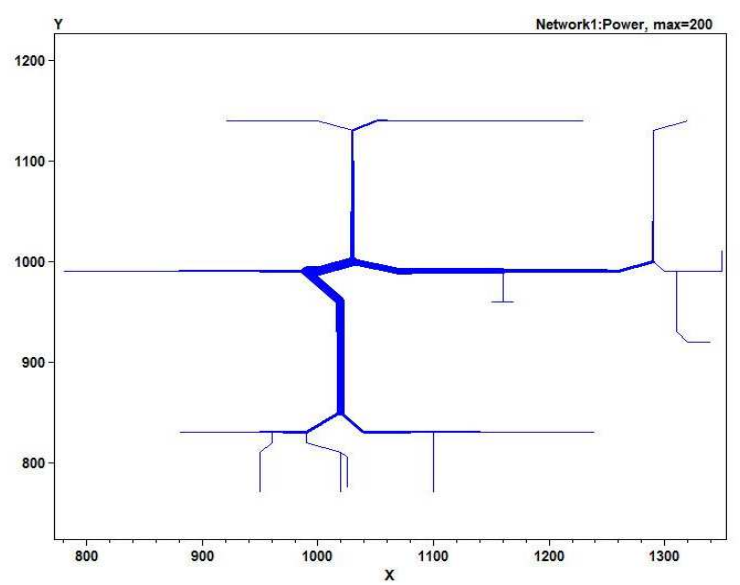

Fig. 10. Graphical load flow of Network1 at 18:00 on a weekday in Feb. 2015.

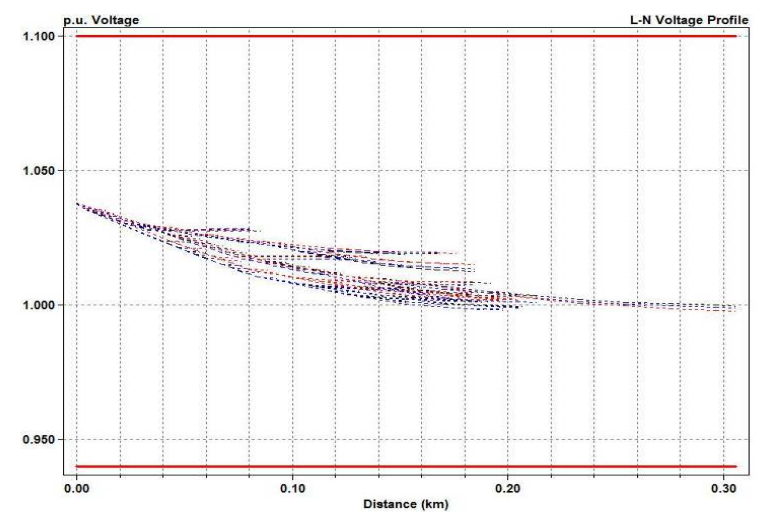

Fig. 11. Diagram of voltage profile against the distance from substation.

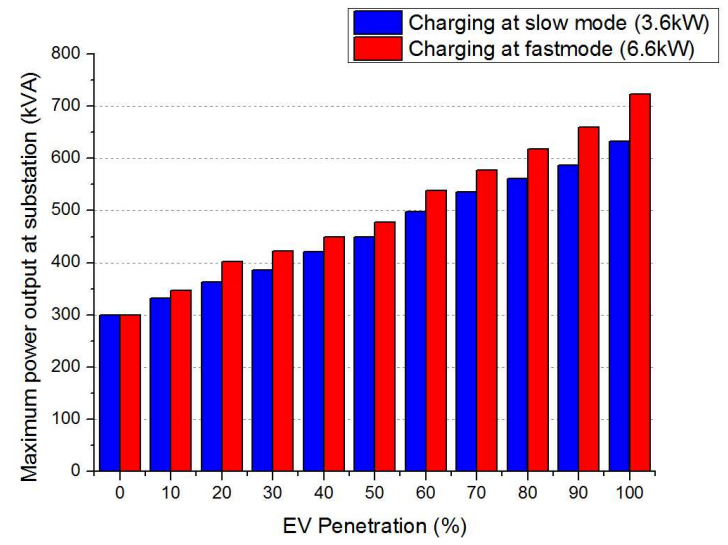

Fig. 12. Thermal issue in Network1 at different EV penetration levels. 


\section{A. Thermal Issues}

Time series simulation of Network1 in Fig. 12 shows that when the EV penetration level is over $60 \%$ at slow charging mode, the transformer will suffer thermal issue (breaching 500kVA capacity). While for fast charging mode, when the penetration level reaches $50 \%$, thermal problem occurs. Similar result can also be observed in Network 2 power flow results.

\section{B. Voltage Issues}

Even at the highest overall load (load and EV) profile, the minimum voltages simulated at each cut-off of properties show no voltage drop issue, i.e. all node voltages are above 0.94p.u..

\section{Automated Load Transferring}

Network reconfiguration in particular automated load transferring (ALT) is implemented to reduce losses of the network as an option to eliminate network thermal constraints and voltage drop issues. Results after ALT are compared with those without ALT technique. Equations of the mathematical problem to describe the system load flow with constraints such as thermal limits and voltage limits are presented [6, 8]. In this paper, network reconfiguration is aimed to reduce entire power losses and balance the loading of each network. Objective function is given as (3):

$$
f=\min \left\{P_{\text {loss }}=\sum_{i=1}^{m} I_{i}^{2} \times R_{i}+\sum_{j=1}^{n} I_{j}^{2} \times R_{j}\right\}
$$

where $m$ is the number of branches in network $1, n$ is the number of branches in network $2, I_{i}$ and $I_{j}$ are the currents in $i^{\text {th }}$ and $j^{\text {th }}$ branches, $R_{i}$ and $R_{j}$ are the corresponding branch resistances.

Voltages at service cut-out shall not exceed by $10 \%$ of $230 \mathrm{~V}$ (single-phase) and lower by $6 \%$. Thermal constraint is that maximum cyclic loading at the substation transformer is $100 \%$ of its rated capacity. According to the thermal issue quantified in part $\mathrm{A}$, a certain case, when the $\mathrm{EV}$ penetration is $60 \%$ in both networks and charging at fast mode, is investigated to understand how ALT can benefit the entire network in capacity improvement and loss reduction. The overall loss of two networks is $14.8 \mathrm{~kW}$. The ALT result indicates to move one of the school from Network 2 to Network 1. However, only a low amount of loss is reduced in this case. The headroom of EV penetration is increased for Network 2 but not quantified here. This result suggests that part of the loads in Network 2 can be moved to Network 1 to reduce overall loss. Other scenarios will be investigated in the future to consider weekend load profiles and EV charging profiles.

\section{CONCLUSION}

LV distribution network modelling used to be done empirically and to some extent crudely for network planning. Modelling at detailed level is not sufficient for new scenarios considering renewable power integration and emerging electric loads e.g. EVs. This paper contributes to detail modelling by implementing a simulation platform for the above purposes. Network model, load profile models, EV charging models are all included to a level of accurately representing the real-life systems. For two case study networks, under different EV charging scenarios, issues such as thermal constraints and voltage drops are presented with ALT as one potential solution. Objective function considered for the moment is the conventional loss reduction. With more options available such as Economy 7 meters for variable electricity import tariffs, economic objectives can be incorporated. Other solutions like EV charging and particularly discharging management are also seen as the ways forward. To fulfill flexible yet smart operations, future works including requirement for $\mathrm{CBs}$ and automation, infrastructure allowing EV discharging, and protection schemes are challenging prerequisite for Smart Grid realization.

\section{ACKNOWLEDGMENT}

The authors wish to thank Western Power Distribution plc. for their support to realize the production of this paper.

\section{REFERENCES}

[1] Feed-in tariffs: get money for generating your own electricity, https://www.gov.uk/feed-in-tariffs/overview, accessed July 2016.

[2] Public attitudes towards electric vehicles: 2016, https://www.gov.uk/government/statistics/public-attitudes-towardselectric-vehicles-2016, accessed July 2016.

[3] H. Rahimi-Eichi, U. Ojha, F. Baronti, and M. Y. Chow, "Battery Management System: An Overview of Its Application in the Smart Grid and Electric Vehicles," IEEE Industrial Electronics Magazine, vol. 7, pp. 4-16, 2013.

[4] J. Druitt and W.-G. Früh, "Simulation of demand management and grid balancing with electric vehicles," Journal of Power Sources, vol. 216, pp. 104-116, 2012.

[5] T. Logenthiran, D. Srinivasan, and T. Z. Shun, "Demand Side Management in Smart Grid Using Heuristic Optimization," IEEE Transactions on Smart Grid, vol. 3, pp. 1244-1252, 2012.

[6] E. A. Bueno, C. Lyra, and C. Cavellucci, "Distribution network reconfiguration for loss reduction with variable demands," in Transmission and Distribution Conference and Exposition: Latin America, 2004 IEEE/PES, 2004, pp. 384-389.

[7] D. Montenegro, M. Hernandez, and G. A. Ramos, "Real time OpenDSS framework for distribution systems simulation and analysis," in Transmission and Distribution: Latin America Conference and Exposition (T\&D-LA), 2012 Sixth IEEE/PES, 2012, pp. 1-5.

[8] D. Fei and K. Loparo, "Hierarchical decentralized network reconfiguration for smart distribution systems-Part I: Problem formulation and algorithm development," in 2015 IEEE Power \& Energy Society General Meeting, 2015, pp. 1-1.

[9] M. E. Baran and F. F. Wu, "Network reconfiguration in distribution systems for loss reduction and load balancing," IEEE Transactions on Power Delivery, vol. 4, pp. 1401-1407, 1989.

[10] F. Ji-Yuan, Z. Lan, and J. D. McDonald, "Distribution network reconfiguration: single loop optimization," IEEE Transactions on Power Systems, vol. 11, pp. 1643-1647, 1996.

[11] D. Fei and K. Loparo, "Hierarchical decentralized network reconfiguration for smart distribution systems-Part II: Applications to test systems," in 2015 IEEE Power \& Energy Society General Meeting, 2015, pp. 1-1.

[12] C.-T. Su, C.-F. Chang, and J.-P. Chiou, "Distribution network reconfiguration for loss reduction by ant colony search algorithm," Electric Power Systems Research, vol. 75, pp. 190-199, 2005.

[13] L. M. O. Queiroz and C. Lyra, "Adaptive Hybrid Genetic Algorithm for Technical Loss Reduction in Distribution Networks Under Variable Demands," IEEE Transactions on Power Systems, vol. 24, pp. 445-453, 2009.

[14] I. Richardson, M. Thomson, D. Infield, and C. Clifford, "Domestic electricity use: A high-resolution energy demand model," Energy and Buildings, vol. 42, pp. 1878-1887, 2010.

[15] J. Quir, T. s, L. F. Ochoa, and B. Lees, "A statistical analysis of EV charging behavior in the UK," in Innovative Smart Grid Technologies Latin America (ISGT LATAM), 2015 IEEE PES, 2015, pp. 445-449.

[16] https://www.nissan.co.uk/, accessed July 2016. 